\title{
A NEW BASIS FOR DISCRETE ANALYTIC POLYNOMIALS
}

\author{
DORON ZEILBERGER
}

(Received 9 January 1976; revised 13 April 1976)

\begin{abstract}
A new basis $\left\{\pi_{k}(z)\right\}_{k=0}^{\infty}$ for discrete analytic polynomials is presented for which the series $\sum_{k=0}^{x} a_{k} \pi_{k}(z)$ converges absolutely to a discrete analytic function in the upper right quarter lattice whenever $\overline{\lim }\left|a_{k}\right|^{1 / k}=0$.
\end{abstract}

\section{Introduction}

Let $Z$ be the group of integers and consider functions

$$
f: Z \times Z \rightarrow \mathbf{C}
$$

such that

$$
f(x, y)+i f(x+1, y)-f(x+1, y+1)-i f(x, y+1)=0
$$

for every $(x, y) \in Z \times Z$. Such functions are termed discrete entire. If (1.1) holds only for $(x, y) \in G, G \subset Z \times Z$, then we say that $f$ is discrete analytic in $G$.

Discrete analytic functions were introduced by Ferrand (1944) and the theory was developed by Duffin (1956) and others.

Duffin (1956) introduced the following basis for discrete analytic polynomials

$$
\rho_{k}(z)=\left.\frac{d^{k}}{d t^{k}}\left\{\left(\frac{2+t}{2-t}\right)^{x}\left(\frac{2+i t}{2-i t}\right)^{y}\right\}\right|_{t=0}
$$

$(z=x+i y)$, which he called pseudo-powers.

Each $\rho_{k}(z)$ is a discrete entire function and a polynomial of degree $k$ in $(x, y)$. Duffin $(1956)$ showed that every discrete analytic polynomial can be expressed as a linear combination of these pseudo-powers.

Duffin and Peterson (1968) introduced an analogue of the McClaurin series in terms of these pseudo-powers. However, their analogue has the dis- 
advantage that the convergence of $\Sigma_{0}^{\infty} a_{n} \xi^{n}$ on $\mathbf{C}$ does not ensure the convergence of $\sum_{0}^{\infty} a_{n} \rho_{n}(z)$ on $Z \times Z$. In fact they showed that $\sum_{0}^{\infty} a_{n} \rho_{n}(z)$ converges on the whole lattice $Z \times Z$ only if

$$
\varlimsup \lim \left(\left|a_{n}\right| n !\right)^{1 / n}<2 .
$$

In Section 2 other "reasonable" bases for discrete analytic polynomials will be considered. These will be called systems of pseudo-powers, and it will be shown that the above drawback of Duffin's basis $\left\{\rho_{n}(z)\right\}$ as regards the convergence of $\sum a_{n} \rho_{n}(z)$ cannot be removed by using other systems of pseudopowers.

On the other hand, we shall construct a system of pseudo-powers $\left\{\pi_{k}(z)\right\}_{0}^{\infty}$ such that $\Sigma_{0}^{\infty} a_{k} \pi_{k}(z)$ converges absolutely on the quarter lattice $Z^{+} \times$ $Z^{+}=\{(x+i y) ; x$ and $y$ integers, $x \geqq 0, y \geqq 0\}$ whenever $\sum_{0}^{\infty} a_{k} \xi^{k}$ converges on the entire plane. (The divergence of $\Sigma_{0}^{\infty}\left(2^{n} / n !\right) \rho_{n}(1,0)$ shows that this property is not enjoyed by the Duffin-Peterson series.)

In Section 3 we shall consider the existence and uniqueness of the expansion $\sum_{0}^{\infty} a_{k} \pi_{k}(z)$. The discrete analogue of 'multiplication by $z$ ' corresponding to the above basis will also be dealt with.

In Section 4 , we discuss the lattice $Z_{h}^{+} \times Z_{h}^{+}$where $Z_{h}^{+}=h Z^{+}, h>0$ and show that if $\left\{\pi_{k}^{h}(z)\right\}_{0}^{\infty}$ is the corresponding basis then

$$
\sum_{0}^{\infty} a_{k} \pi_{k}^{h}(z) \rightarrow \sum_{0}^{\infty} a_{k} z^{k}
$$

when $h \downarrow 0$ along a sequence for which $z \in Z_{h} \times Z_{h}$, provided $\Sigma_{0}^{\infty} a_{k} \xi^{k}$ is an entire function of exponential type.

The analogous problem of representing monodiffric functions (that is functions satisfying

$$
(i-1) f(x, y)-i f(x+1, y)+f(x, y+1) \equiv 0)
$$

by a series of polynomials was considered by Atadžanov (1974).

\section{The new basis}

Definition. A basis $\left\{p_{n}(z)\right\}_{0}^{\infty}$ for the discrete analytic polynomials is called a system of pseudo-powers if the following properties are satisfied:

(A1) $p_{n}(0)=0$ for every $n>0$

(A2) $\left\{p_{n}(z)\right\}$ satisfies the binomial identity

$$
p_{n}\left(z_{1}+z_{2}\right)=\sum_{k=0}^{n}\left(\begin{array}{l}
n \\
k
\end{array}\right) p_{k}\left(z_{1}\right) p_{n-k}\left(z_{2}\right)
$$


(A3) $p_{0} \equiv 1$ and for $n \geqq 0 p_{n}(z)=z^{n}+\tilde{p}_{n-1}(x, y)$ where $\tilde{p}_{n-1}$ is a polynomial of degree $\leqq n-1$.

It is readily checked that Duffin's basis $\left\{\rho_{n}(z)\right\}$ constitutes a system of pseudo-powers. On the other hand, Duffin's basis fails to satisfy the following:

$$
\begin{gathered}
\sum_{0}^{\infty} a_{n} p_{n}(z) \text { converges absolutely for every } z \in Z \times Z \\
\text { if } \sum_{0}^{\infty} a_{n} \xi^{n} \text { converges in the whole } \xi \text {-plane. }
\end{gathered}
$$

One may ask: Does there exist a system of pseudo-powers satisfying (*)? That no such system exists follows from the next lemma.

Lemma 2.1. Let $\left\{p_{k}\right\}$ be any system of pseudo-powers. Then there exists a point $z_{0}$ in the half lattice $\{x+i y, y \geqq 0\}$ and a complex number $\zeta_{0}$ such that

$$
\sum_{0}^{\infty} \frac{\zeta_{0}^{k}}{k !} p_{k}\left(z_{0}\right) \text { fails to converge absolutely. }
$$

Proof. Suppose that the statement is false, i.e., there exists a system of pseudo-powers $\left\{p_{k}\right\}$ such that

$$
e(\zeta ; z)=\sum_{k=0}^{\infty} \frac{\zeta^{k}}{k !} p_{k}(z)
$$

converges absolutely for every point in the half lattice and for every complex number $\zeta$. Then, for every such $z, e(\zeta, z)$ is an entire function in $\zeta$ and by (A2)

$$
\begin{aligned}
e\left(\zeta ; z_{1}\right) e\left(\zeta ; z_{2}\right) & =\left(\sum_{0}^{\infty} \frac{\zeta^{k}}{k !} p_{k}\left(z_{1}\right)\right)\left(\sum_{0}^{\infty} \frac{\zeta^{r}}{r !} p_{r}\left(z_{2}\right)\right) \\
& =\sum_{0}^{\infty} \frac{\zeta^{n}}{n !}\left[\sum_{0}^{n}\left(\begin{array}{l}
n \\
k
\end{array}\right) p_{k}\left(z_{1}\right) p_{n-k}\left(z_{2}\right)\right] \\
& =\sum_{0}^{\infty} \frac{\zeta^{n}}{n !} p_{n}\left(z_{1}+z_{2}\right)=e\left(\zeta ; z_{1}+z_{2}\right) .
\end{aligned}
$$

Thus $e(\zeta ; x+i y)=f(\zeta)^{x} g(\zeta)^{y}$ where $f(\zeta)=e(\zeta ; 1), g(\zeta)=e(\zeta ; i)$.

Since $e(\zeta ; z)$ is discrete analytic in the upper half lattice $(1.1)$ must be satisfied there:

$$
f(\zeta)^{x} g(\zeta)^{y}\{1+i f(\zeta)-f(\zeta) g(\zeta)-i g(\zeta)\}=0
$$

Thus

$$
g(\zeta)=\frac{1+i f(\zeta)}{f(\zeta)+i}
$$


and

$$
e(\zeta ; x+i y)=f(\zeta)^{x}\left(\frac{1+i f(\zeta)}{f(\zeta)+i}\right)^{y}
$$

Since $e(\zeta ; z)$ is entire in $\zeta$ for each fixed $z$ in the half lattice and in particular for $z=1,-1, i$ we see that $f(\zeta), 1 / f(\zeta)$ and $(1+i f(\zeta)) /(f(\zeta)+i)$ are entire. But this implies that $f(\zeta)$ is entire and excludes the values 0 and $-i$. By the "little" Picard theorem [Rudin (1966), p. 324] this is too much to ask from a non-constant entire function. Evidently $f(\zeta)$ cannot be constant and so we arrive at a contradiction and the lemma is proved.

We saw that there is no system of pseudo-powers satisfying $(*)$. The next theorem will demonstrate a system of pseudo-powers satisfying the following weaker property.

(A4) $\sum_{0}^{\infty} a_{n} p_{n}(z)$ converges absolutely for every

$$
z \in Z^{+} \times Z^{+}=\{x+i y ; x \geqq 0, y \geqq 0\} \quad \text { if } \quad \sum_{0}^{\infty} a_{n} \xi^{n}
$$

converges in the whole $\xi$-plane.

The divergence of $\Sigma\left(2^{n} / n !\right) \rho_{n}(1)$ shows that Duffin's basis does not satisfy (A4).

THEOREM 2.2. The sequence of functions

$$
\begin{array}{rl}
\pi_{k}(x, y)=\frac{d^{k}}{d \zeta^{k}}\left\{\left[(1+i) e^{\zeta /(i+1)}-i\right]^{x}\left[(1-i) e^{-\zeta /(1+i)}+i\right]^{y}\right\} & \left.\right|_{\zeta=0} \\
k & k=0,1,2, \cdots
\end{array}
$$

constitutes a system of pseudo-powers satisfying (A4).

PROOF. The discrete analyticity of $\pi_{k}(x, y)$ is readily checked. (A1) is trivial, while (A2) follows from Leibnitz' formula. Also, by a straightforward computation

$$
\pi_{k+1}(x, y)=\frac{1}{1+i}\left\{(x-y) \pi_{k}(x, y)+i x \pi_{k}(x-1, y)+i y \pi_{k}(x, y-1)\right\} .
$$

Since $\pi_{0}(x, y) \equiv 1$ it follows by induction that each $\pi_{k}(x, y)$ is a polynomial of degree $k$ and that (A3) holds. Since Duffin (1956) showed that the dimension of the space of discrete analytic polynomials of degree $\leqq k$ is $k+1$, it follows that $\left\{\pi_{r}\right\}_{0}^{k}$ is a basis for the discrete analytic polynomials of degree $\leqq k$ and consequently that $\left\{\pi_{k}\right\}_{0}^{\infty}$ is a basis for the discrete analytic polynomials. Thus $\left\{\pi_{k}\right\}$ is a system of pseudo-powers. 
Now, let us note that for a fixed $z=x+i y \in Z^{+} \times Z^{+}$

$$
e(\zeta ; x+i y)=\sum_{0}^{\infty} \pi_{k}(x, y) \frac{\zeta^{k}}{k !}=\left[(1+i) e^{\zeta /(i+1)}-i\right]^{\times}\left[(1-i) e^{-\zeta /(1+i)}+i\right]^{y} .
$$

Since $x$ and $y$ are non-negative integers, the right hand side is an entire function of exponential type and the Taylor coefficients being $\pi_{k}(x, y) / k$ ! you have (Boas (1954), p. 11) that there exist constants $C$ and $T$ (depending on $(x, y))$ such that

$$
\left|\pi_{k}(x, y)\right| \leqq C T^{k}
$$

Thus $\sum_{0}^{\infty} a_{k} \pi_{k}(x, y)$ converges absolutely whenever $\overline{\lim }\left|a_{k}\right|^{1 / k}=0$, since $\sum_{0}^{x} a_{k} T^{k}$ does. This holds for every $(x, y) \in Z^{+} \times Z^{+}$and it follows that $\left\{\pi_{k}\right\}$ is a system of pseudo-powers satisfying (A4).

By Theorem (2.2) it follows that whenever $\sum_{0}^{\infty} a_{k} \xi^{k}$ is an entire function, i.e., whenever $\overline{\lim }\left|a_{k}\right|^{1 / k}=0$, then $\Sigma_{0}^{\infty} a_{k} \pi_{k}(z)$ converges to a discrete analytic function in $Z^{+} \times Z^{+}$(substitute in (1.1) and rearrange terms, using the fact that each $\pi_{k}(z)$ is discrete analytic).

Let $\mathscr{A}$ be the algebra of entire functions and let $\mathscr{D}$ be the set of discrete analytic functions on $Z^{+} \times Z^{+}$. Define a mapping

$$
T: \mathscr{A} \rightarrow \mathscr{D}
$$

by

$$
T\left(\sum_{0}^{\infty} a_{n} \xi^{n}\right)=\sum_{0}^{\infty} a_{n} \pi_{n}(z)
$$

Let $\mathscr{F} \subset \mathscr{D}$ be the range of $T . \mathscr{F}$ can be made into an algebra by requiring $T$ to be a homomorphism:

$$
\left(\sum_{0}^{\infty} a_{k} \pi_{k}(z)\right)\left(\sum_{0}^{\infty} b_{k} \pi_{k}(z)\right)=\sum_{n=0}^{\infty}\left(\sum_{k=0}^{n} a_{k} b_{n-k}\right) \pi_{n}(z) .
$$

Thus in our class $\mathscr{F}$, multiplication is defined for every pair $f, g \in \mathscr{F}$. This is an improvement on the multiplication in the Duffin-Peterson class,

$$
\mathscr{F}_{D P}=\left\{\sum_{0}^{\infty} a_{n} \rho_{n}(z) ; \varlimsup \overline{\lim }\left(\left|a_{n}\right| n !\right)^{1 / n}<2\right\}
$$

which is only defined on a subset of $\mathscr{F}_{D P} \times \mathscr{F}_{D P}$. In particular $\exp f$ is well defined in our class:

$$
\exp \left(\sum_{0}^{\infty} a_{k} \pi_{k}(z)\right)=T\left(\exp \left(\sum_{0}^{\infty} a_{k} \xi^{k}\right)\right) .
$$




\section{Existence and uniqueness of Taylor expansion}

Formula (2.2) motivates the following analogue for the continuous "multiplication by $z "$

$$
f(x, y)=\frac{1}{1+i}\{(x-y) f(x, y)+i x f(x-1, y)+i y f(x, y-1)\} .
$$

It is readily checked that if $f$ is discrete analytic, then so is $z f$ and, by (2.2)

$$
z \pi_{k}=\pi_{k+1} \quad z e(\xi ; x+i y)=\frac{d}{d \xi} e(\xi ; x+i y) .
$$

Let us restrict attention to $\mathscr{D}$, the class of discrete analytic functions on $Z^{+} \times$ $Z^{+}$. It was shown in Zeilberger (to appear) that each $f \in \mathscr{D}$ is uniquely determined by the pair of formal power series $\left(\phi_{f}, \psi_{f}\right)$ where

$$
\phi_{f}(X)=\sum_{x=0}^{\infty} f(x, 0) X^{x}, \quad \psi_{f}(Y)=\sum_{y=0}^{\infty} f(0, y) Y^{y},
$$

and we write $f=\left(\phi_{f}, \psi_{f}\right)$.

Since $\not f f(x, 0)=1 /(1+i)\{x f(x, 0)+i x f(x-1,0)\}$

$$
\begin{aligned}
\sum_{x=0}^{\infty}\left\{f f(x, 0) X^{x}\right. & =\frac{1}{1+i} \sum_{x=0}^{\infty} x(f(x, 0)+i f(x-1,0)) X^{x} \\
& =\frac{X}{1+i} \frac{d}{d X}\left[(1+i X) \phi_{f}(X)\right] .
\end{aligned}
$$

Similarly

$$
\sum_{y=0}^{\infty} z f(0, y) Y^{y}=\frac{Y}{1+i} \frac{d}{d Y}\left[(i Y-1) \psi_{f}(Y)\right]^{\cdot}
$$

So the operation of $z$ in terms of formal power series is

$(3.2)\left(\phi_{f}, \psi_{f}\right) \rightarrow \frac{1}{1+i}\left(X \frac{d}{d X}\left[(1+i X) \phi_{f}(X)\right], \quad Y \frac{d}{d Y}\left[(i Y-1) \psi_{f}(Y)\right]\right)$.

Thus $\not f \equiv 0$ iff

$$
\phi_{f}(X)=\frac{C}{1+i X} ; \quad \psi_{f}(Y)=\frac{C}{1-i Y} .
$$

(The constants agree since $\phi_{f}(0)=f(0,0)=\psi_{f}(0)$.) So, unfortunately, has a non-trivial kernel.

Clearly, $f f(0)=0$ for every function $f$ discrete analytic in $Z^{+} \times Z^{+}$. Let $g \in \mathscr{D}, g(0)=0$ then $f \in \mathscr{D}$ given by 


$$
\begin{aligned}
& \phi_{f}(X)=\frac{1+i}{1+i X} \int \frac{\phi_{g}(X)}{X} d X=\frac{1+i}{1+i X}\left[\sum_{1}^{\infty} \frac{g(x, 0) X^{x}}{x}+C\right] \\
& \psi_{f}(Y)=\frac{1+i}{1-i Y} \int \frac{\phi_{g}(Y)}{Y} d Y=\frac{1+i}{1-i Y}\left[\sum_{1}^{\infty} \frac{g(0, y) Y^{y}}{y}+C\right]
\end{aligned}
$$

solves $z f=g$.

We have thus obtained

THEOREM 3.1. The operator

$$
\ddot{z}: \mathscr{D} \rightarrow \mathscr{D}
$$

has range $\{f \in \mathscr{D} ; f(0,0)=0\}$ and kernel $\left\{C f_{0}\right\}$ where $f_{0} \in \mathscr{D}$ is given by

$$
\phi_{f_{0}}=\frac{1}{1+i X} ; \quad \psi_{f_{0}}=\frac{1}{1-i Y} .
$$

Let us consider the class $\mathscr{F} \subset \mathscr{D}$ defined at the end of Section 2. It is not yet known whether the inclusion $\mathscr{F} \subset \mathscr{D}$ is proper or not; i.e., whether every discrete analytic function on $Z^{+} \times Z^{+}$possesses a discrete Taylor expansion

$$
f(z)=\sum_{k=0}^{\infty} a_{k} \pi_{k}(z)
$$

Theorem (3.1) implies that even if such a representation exists it need not be unique. However if attention is restricted to the class

$$
\mathscr{F}_{e}=\left\{\sum_{0}^{\infty} a_{k} \pi_{k}(z) ; \quad \overline{\lim }\left(k !\left|a_{k}\right|\right)^{1 / k}<\infty\right\}
$$

then the representation (3.3) is unique, as follows from the following

THEOREM 3.2. If $\sum_{0}^{\infty} a_{k} \pi_{k}(z) \equiv \overline{0}$ in $Z^{+} \times Z^{+}$and $\overline{\lim }\left(k !\left|a_{k}\right|\right)^{1 / k}<\infty$ then $a_{k}=0$ for every $k$.

Proof. By definition (2.1)

$$
\pi_{k}(x, y)=\frac{k !}{2 \pi i} \int_{\Gamma} \frac{\left[(1+i) e^{\zeta /(1+i)}-i\right]^{x}\left[(1-i) e^{-\zeta /(1+i)}+i\right]^{y}}{\zeta^{k+1}} d \zeta
$$

where $\Gamma$ is any contour surrounding 0 . So,

$$
\begin{aligned}
f(z) & =\sum_{0}^{\infty} a_{k} \pi_{k}(x, y) \\
& =\frac{1}{2 \pi i} \int_{\Gamma}\left(\sum \frac{k ! a_{k}}{\zeta^{k+1}}\right)\left[(1+i) e^{\zeta /(1+i)}-i\right]^{x}\left[(1-i) e^{-\zeta /(1+i)}+i\right]^{y} d \zeta
\end{aligned}
$$


for any contour $\Gamma$ for which

$$
f_{B}(\zeta)=\sum_{k=0}^{\infty} \frac{k ! a_{k}}{\zeta^{k+1}}
$$

is defined. $f_{B}(\zeta)$ is the Borel transform of

$$
f^{c}(\zeta)=\sum_{k=0}^{\infty} a_{k} \zeta^{k}
$$

and $f_{B}(\zeta)$ converges for $|\zeta| \geqq$ type $f^{c}$ (see Boas (1954), p. 73). Thus

$$
f(z)=\frac{1}{2 \pi i} \int_{\Gamma} f_{B}(\zeta)\left[(1+i) e^{\zeta /(1+i)}-i\right]^{x}\left[(1-i) e^{-\zeta /(1+i)}+i\right]^{y} d \zeta
$$

and for some constant $M$

$$
|f(x, 0)| \leqq C M^{x}
$$

and $\phi_{f}(t)=\Sigma_{0}^{\infty} f(x, 0) t^{x}$ converges in the disc $|t|<1 / M$. We have then

$$
\begin{aligned}
\phi_{f}(t) & =\sum_{x=0}^{\infty} f(x, 0) t^{x}=\frac{1}{2 \pi i} \int_{\Gamma} f_{B}(\zeta) \sum_{0}^{\infty}\left[(1+i) e^{\zeta /(1+i)}-i\right]^{x} t^{x} \\
& =\frac{1}{2 \pi i} \int_{\Gamma} \frac{f_{B}(\zeta) d \zeta}{1-\left[(1+i) e^{\zeta /(1+i)}-i\right] t} .
\end{aligned}
$$

The right hand side defines an analytic function in any region in the $t$-plane for which the denominator of the integrand does not vanish in a neighborhood of $\Gamma$ in the $\zeta$-plane. In particular, this includes a neighborhood of the point $i$ in the $t$-plane. Thus for any discrete analytic function of class $\mathscr{F}_{e}$

$$
\phi_{f}(t)=\sum_{x=0}^{\infty} f(x, 0) t^{x}
$$

whose radius of convergence is in general smaller than 1 , can be analytically continued through the boundary of the circle of convergence to a neighborhood of $t=i$.

Now $\Sigma_{0}^{\infty} a_{k} \pi_{k}(z) \equiv 0$ implies $a_{0}=0$ and

$$
z\left(\sum_{1}^{\infty} a_{k} \pi_{k-1}(z)\right)=0 \text {. }
$$

Let $g_{1}(z)=\sum_{1}^{\infty} a_{k} \pi_{k-1}(z)$. Then $g_{1} \in \mathscr{F}_{e}$ and hence $\phi_{g_{1}}(t)$ can be analytically continued to a neighborhood of $t=i$. But $z g_{1} \equiv 0$ implies, by Theorem 3.1, that $\phi_{\mathrm{B}_{1}}(t)=C /(1+i t)$ for some constant $C$. This forces $C=0$ for, otherwise $\phi_{81}$ would have a pole at $t=i$. Thus,

$$
g_{1}(z)=\sum_{1}^{\infty} a_{k} \pi_{k-1}(z) \equiv 0 \quad \text { and } \quad a_{1}=0 .
$$


Continuing inductively we get that $a_{k}=0$ for every $k$ and the theorem is proved.

\section{Limiting behavior as $h \downarrow 0$}

Let $h>0$. For the lattice of mesh size $h$

$$
Z_{h} \times Z_{h}=\{(h m, h n) ; m, n \in Z\}
$$

discrete analyticity is defined by

$$
F(x, y)+i F(x+h, y)-F(x+h, y+h)-i F(x, y+h)=0 .
$$

The above discussion carries over to discrete analytic functions for such lattices (all it amounts to is a change of scale). Now we have the basis

$$
\pi_{k}^{h}(x, y)=\left.\frac{d^{k}}{d \zeta^{k}}\left\{\left[(1+i) e^{\zeta h /(1+i)}-i\right]^{x / h}\left[(1-i) e^{-\zeta h /(1+i)}+i\right]^{y / h}\right\}\right|_{\zeta=0}
$$

And for discrete analytic functions on the lattice $Z_{h} \times Z_{h}$ the exponential function is

$$
e_{h}(x, y)=\sum_{k=0}^{\infty} \pi_{k}^{h}(x, y) \frac{\zeta^{k}}{k !}=\left[(1+i) e^{\zeta h /(1+i)}-i\right]^{x / h}\left[(1-i) e^{-\zeta h /(1+i)}+i\right]^{y / h}
$$

Now as $h \downarrow 0$

$$
\left[(1+i) e^{\zeta h /(1+i)}-i\right]^{1 / h} \rightarrow e^{\zeta} \quad\left[(1-i) e^{-\zeta h /(1+i)}+i\right]^{1 / h} \rightarrow e^{i \zeta} .
$$

So $e_{h}(x, y) \rightarrow e^{5(x+i y)}$ and consequently

$$
\pi_{k}^{h}(z) \rightarrow z^{k} \quad \text { as } \quad h \downarrow 0 .
$$

Suppose $\left|a_{n}\right| \leqq C \zeta_{0}^{n} / n$ ! for some constants $C$ and $\zeta_{0}$, by dominated convergence

$$
f^{h}(z)=\sum_{0}^{\infty} a_{k} \pi_{k}^{h}(z) \rightarrow \sum_{0}^{\infty} a_{k} z^{k}
$$

as $h \downarrow 0$. We obtained

LEMMA 4.1. If $\overline{\lim }\left(\left|a_{k}\right| k !\right)^{1 / k}<\infty$ then $f^{h}(z) \rightarrow f^{c}(z)=\sum_{0}^{\infty} a_{k} z^{k}$ along $a$ sequence $h \downarrow 0$ for which $z \in Z_{h}^{+} \times Z_{h}^{+}$.

\section{Acknowledgement}

The author wishes to thank Professor Harry Dym, Dr. Isidore Shapiro and Mrs. Miriam Drory for helpful comments and discussions. He would also like to thank the referee for bringing Atadžanov's paper to his attention. 


\section{References}

B. A. Atadzanov (1974), 'Representation of analytic functions of a discrete complex variable by means of basis elements'. Metričeskie Voprosy Teor. Funkciu, Otobražaniu 5, 13-20 (Russian).

R. P. Boas, Jr (1954), Entire Functions (Academic Press, New York).

R. J. Duffin (1956), 'Basic propertiess of discrete analytic functions', Duke Math. J. 23, 335-363.

R. J. Duffin and Elmore L. Peterson (1968), 'The discrete analogue of a class of entire functions'. J. Math. Anal. Appl. 21, 619-642.

J. Ferrand (1944), 'Fonctions préharmonique et fonctions préholomorphes', Bull. Sci. Math. 68, $152-180$.

W. Rudin (1966), Real and Complex Analysis (McGraw-Hill, New York).

D. Zeilberger (to appear), 'A new approach to the theory of discrete analytic functions'. J. Math. Anal. Appl.

Department of Mathematics,

Weizmann Institute of Science,

Rehovot, Israel. 